\title{
Estimation of Soluble CD14 Level in Saliva of Patients with Chronic Periodontitis \& Its Correlation with Periodontal Health Status \& Disease Severity.
}

\author{
${ }^{1}$ Sarah E.H Al-Karawi, ${ }^{2}$ Maha Sh. Al-Rubaie, \\ ${ }^{I}$ B.D.S.M.Sc. student. Department of Periodontics. College of Dentistry. University of Baghdad. Baghdad-Iraq. \\ ${ }^{2}$ B.D.S., M.Sc. Assistant Professor. Department of Periodontics. College of Dentistry. University of Baghdad. \\ Baghdad-Iraq.
}

\begin{abstract}
:
Background: Cluster of differentiation 14 (CD14) is a host cell recognition receptor, that found on the cell membrane of monocytes/macrophages and neutrophils or soluble in saliva and other body fluids, and is involved in the recognition of microbial-associated patterns. It acts on the cell membrane by retaining lipopolysaccharide (LPS) and other bacterial ligands and facilitating ligand-toll-like receptor (TLR) interaction, resulting in the release of cytokines from the inflammatory cells. This study was conducted to assess the level of soluble CDI4 in saliva of patients with chronic periodontitis of different severities and healthy subjects and determine its correlation with clinical periodontal parameters.

Materials \& Methods: A total of 45 chronic periodontitis patients, age ranged (35-50) years old, divided into three subgroups according to different severities (Mild=15, Moderate $=15$ and Severe $=15$ ). And the control group comprised 15 apparently- healthy volunteers. Unstimulated whole saliva samples were collected to determine levels of soluble CD14 in saliva by enzyme-linked immune-sorbent assay (ELISA). Clinical periodontal parameters were recorded at four sites per tooth including plaque index, gingival index, bleeding on probing, probing pocket depth and clinical attachment level.

Results: A highly significant difference $(p<0.01)$ was found for salivary sCD14 levels among the three subgroups (the severe group present the higher median), Furthermore, Spearman's correlation analysis showed a highly significant strong correlations $(p<0.01)$ between salivary sCD14 levels and each of (probing pocket depth\& clinical attachment level), and non-significant correlation between salivary sCD14 level with plaque, gingival \& bleeding on probing indices.

Conclusion: sCD14 may play a crucial role in periodontal disease pathogenesis and severity. Salivary sCD14 level may reflect the extent of the disease and so it may predict future disease progression.

Key Words: soluble CD14, chronic periodontitis, saliva.
\end{abstract}

\section{Introduction}

Periodontitis is defined as an inflammatory disease of the supporting tissues of the teeth caused by specific microorganisms or groups of specific microorganisms, resulting in progressive destruction of the periodontal ligament and alveolar bone with increased probing pocket depth formation, gingival recession, or both. The clinical feature that distinguishes periodontitis from gingivitis is the presence of clinically detectable attachment loss. This loss often is accompanied by periodontal pocket formation and changes in the density and height of subjacent alveolar bone ${ }^{(1)}$. In some cases, recession of the marginal gingiva may accompany attachment loss, thus masking ongoing disease progression if only probing depth measurements are taken without measurements of clinical attachment levels ${ }^{(2)}$. Chronic periodontitis usually has slow to moderate rates of progression, but may have periods of rapid progression ${ }^{(3)}$. The disease is classified according to severity as slight or mild: 1 to $2 \mathrm{~mm}$ moderate: 3 to $4 \mathrm{~mm}$, or severe: $\geq 5 \mathrm{~mm}$ based on the amount of clinical attachment loss. $(1 ; 3)$

Cluster of differentiation 14 (CD14) is a serum/cell surface glycoprotein; and it is a pattern recognition receptor(PRR) ${ }^{(4,5)}$. Expressed on the surface of various cells, including peripheral blood monocytes, tissue macrophages ${ }^{(6)}$, neutrophils, and chondrocytes ${ }^{(7)}$, as well as gingival fibroblasts ${ }^{(8)}$. The CD14 can be found in two forms, a membrane-bound (mCD14) ${ }^{(9,10)}$ and a circulating soluble (sCD14) form found in saliva, GCF, serum and urine. ${ }^{(11,12,13,14,15)} \mathrm{sCD} 14$ is slightly smaller than mCD14 and is believed to be the result of enzymatic shedding/cleavage of the membrane form of the receptor CD14 by specific proteases or phospholipases ${ }^{(\mathbf{9})}$ or is directly secreted from intracellular vesicles ${ }^{\left({ }^{(1)}\right)}$ or secreted from salivary glands. sCD14 may be produced by a large number of tissue macrophages or other CD14-expressing tissue cells, like hepatocytes ${ }^{(17)}$ 
Earlier studies showed that sCD14 has the ability to interact withLPS, and those sCD14-LPS complexes can activate human cells that normally lack mCD14, such as epithelial cells, thus triggering an inflammatory response ${ }^{(18,19)}$ Furthermore, a comparative analysis of GCF samples obtained from patients with different periodontal conditions revealed the presence of higher amounts of SCD14 in periodontitis group than in healthy subjects. ${ }^{(\mathbf{1 4})}$ Other Study ${ }^{(\mathbf{2 0})}$ found elevated sCD14 levels in periodontitis and which increased with the severity of periodontal destruction and paralleled by markers of a systemic inflammatory reaction. While limited information is available on the exact contribution of sCD14 to, and its mechanism of action in, the pathogenesis of periodontal disease, it can be speculated that SCD14 plays a significant role because it is detected in elevated amounts in the GCF of patients with periodontitis. ${ }^{(14)}$ Soluble CD14 interacts with epithelial cells that do not normally express endogenous CD14. ${ }^{(21,22)}$ However, it has been proposed that SCD14 may also play a protective role by controlling Gram -ve bacterial infections through its capacity to bind LPS and compete with the mCD14. ${ }^{(23)}$ Moreover, it has been suggested that $\mathrm{SCD} 14$ could be an acute-phase protein, as it can be produced by human hepatocytes and its levels correlate with C-reactive protein and interlukine-6 (IL-6). ${ }^{(24)}$ More recently the innate immune system has enjoyed something of a renaissance, fueled by an explosion in knowledge of pathogen recognition systems such as theTLRs. ${ }^{(\mathbf{1})}$ TLR4 recognizes LPS from Gram -ve bacteria and functions as part of a complex of cell surface molecules, including CD14 and MD-2 (also known as lymphocyte antigen). ${ }^{(25)}$ Interaction of this CD14/TLR4/MD-2 complex with LPS triggers a series of intracellular events, the net result of which is increased production of inflammatory mediators (most notably cytokines) and the differentiation of immune cells (e.g., dendritic cells) for the development of effective immune responses against the pathogens. ${ }^{(1)}$ The sCD14-bound LPS interacts with a receptor complex on the cell surface and initiates one or more host intracellular activation pathways leading to innate host defense mediator production (expression of inflammatory mediators). ${ }^{(26)}$ Besides its function in LPS/cell-wall products signaling, sCD14 might play a role in inflammatory diseases by controlling the immune system level of response. ${ }^{(24)}$ It has been demonstrated that sCD14 is a regulatory factor capable of modulating cellular and humoral immune responses by interacting directly (without LPS) with T- and B- cells, decreasing antigen and mitogen-induced proliferation. ${ }^{(27,28)}$

\section{Materials \&Methods}

Sample population included (60) subjects aged from (35-50) years old. Sample recruited for this study were patients attended to the Department of Periodontics in the Teaching Hospital of College of Dentistry, University of Baghdad seeking periodontal treatment. All subjects enrolled voluntarily in the study after a well explanation about the aim and purposes of the study and gave informed consent to participate in the study in the period between (November, 2012) \& (March, 2013). From each subject, (5ml) of unstimulated whole saliva was harvested; removed particulates by high speed cold centrifugation. The laboratory test was done in the Teaching Laboratories of Baghdad Medical City .

Exclusion criteria included the presence of less than 20 natural teeth, pregnancy and menopause ladies, smokers, Patients received periodontal treatment and /or regular use of anti-inflammatory medication, antibiotics or the use of other medications known to affect the periodontium in the past 3 months. In a cross sectional study, the study group generally were divided into two main groups (chronic periodontitis group \& control group).The chronic periodontitis group was sub divided into three subgroups according to the severity of the disease ${ }^{(3)}$ So the study sample was composed of the following groups:

Group I: Consisted of fifteen (15) patients had mild chronic periodontitis(more than $30 \%$ of the affected sites with CAL range of 1-2 $\mathrm{mm}$ ).

Group II: Consisted of fifteen (15) patients had moderate chronic periodontitis (more than $30 \%$ of the affected sites with CAL range of 3-4 $\mathrm{mm}$ ).

Group III: Consisted of fifteen (15)patients had severe chronic periodontitis (more than $30 \%$ of the affected sites with $\mathrm{CAL} \geq 5 \mathrm{~mm}$ ).

Control Group: Consisted of fifteen (15)healthy volunteers with clinically healthy periodontium.

The periodontal status was evaluated by measurements of the following clinical periodontal parameters: (PLI, GI, BOP, PPD and CAL).Measurements were performed at four sites per tooth for whole mouth excluding the 3rd molars.

\section{Immunological Analysis:}

Enzyme Linked Immuno-Sorbent Assay (ELISA)was used for quantitative determination of sCD14 level in saliva. The work was done in the Immunology Department of Teaching Laboratories of Baghdad Medical City. This assay employs the quantitative sandwich enzyme immunoassay technique. Antibody specific for sCD14 has been precoated onto a microplate. Standards and samples were pipetted into the wells and any sCD14 present was bound by the immobilized antibody. After removing any unbound substances, a biotinconjugated antibody specific for SCD14 was added to the wells. After washing, avidin conjugated Horseradish Peroxidase (HRP) was added to the wells. Following a wash to remove any unbound avidin-enzyme reagent, a 
TMB substrate solution was added to the wells and color develops in proportion to the amount of sCD14 bound in the initial step. The color development was stopped and the intensity of the color was measured.

Statistical Analysis:

Data were calculated and entered into a computerized data base structure. Statistical analysis was done using SPSS software. All parameters were tested for normal distribution using the Shapiro-Wilk test for small sample size. The Mean and SD, t-test, Chi square, and ANOVA test were used to determine the differences between the clinical periodontal parameters. And because the results of sCD14 for each group did not follow a normal distribution, the variables were analyzed using nonparametric methods. To determine the differences sCD14 levels among the groups, the Kruskal-Wallis $\mathrm{H}$ test followed by Mann-Whitney $\mathrm{U}$ test for intergroup comparisons were performed.Correlationsamong sCD14 levels and clinical parameters were assessed using Spearman's correlation coefficient $(r)$. Level of significance for all tests was 0.05 .

\section{Results}

A non-significant statistical difference was observed among chronic periodontitis groups with both PLI and GI and a highly significant statistical difference with BOP as shown in table (1). Inter groups' comparison of the percentages of sites with BOP showed a highly significant difference between the mild \& moderate groups, the mild \& severe groups as well as moderate \& severe groups (table 2).As for the PPD among the chronic periodontitis groups, results showed that severe group presents the higher mean $(4.89053 \pm 0.472738)$ followed by moderate group with a mean value of $(4.64913 \pm 0.404584)$ and lastly the mild group showed the minimum mean value of $(4.50933 \pm 0.451655)$.Using one way ANOVA test a non-significant statistical difference was observed among the chronic periodontitis groups as illustrated in table (3). The mean values of CAL showed that the severe group presents the higher mean $(4.69493 \pm 0.855003)$ followed by moderate group with a mean value of $(2.86413 \pm 0.557106)$ and lastly the mild group showed the minimum mean value of $(2.42480$ \pm 0.614723 ) Using one way ANOVA test a highly statistically significant difference was observed among the chronic periodontitis groups as shown in table (3). Table (4) showed inter groups' comparison for CAL using ttest showed a significant difference between the mild \& moderate groups, a highly significant difference between the mild \& severe groups as well as between the moderate $\&$ severe groups.

Regarding the sCD14 level, table (5) illustrated the median of sCD14 level in the control \& chronic periodontitis groups, it showed a sCD14 level median of (10.359)while the control group showed(5.428). Group comparison between the control \& chronic periodontitis group using the Mann-Whitney test revealed a highly significant difference.Inter group comparison using the Mann-Whitney test showed a highly significant difference between the control \& the mild groups, the control \& the moderate groups as well as the control \& the severe groups as illustrated in table (6)

As for the chronic periodontitis groups the mild group showed a sCD14 level median of (7.392), the moderate group showed (10.359) while the severe group showed (12.798). A Kruskal Wallis test showed a highly statistical significant result in sCD14 level among them as shown in table (7).Inter groups' comparison revealed a statistical significant difference between the mild \& moderate groups \& a highly significant difference between the mild \& severe groups. And a non-significant difference was found between the moderate $\&$ severe groups as shown in table (8).

There was a weak correlation between salivary sCD14 and PLI, GI and BOP parameters with mild, moderate and severe groups as shown in table (9). Table (10)illustrated the correlation between the PPD,CAL parameters and the sCD14 level we noticed a highly significant correlation between the sCD14 level and both PPD and CAL. Figure (1\&2) showed a scatter plot of linear correlation between mean PPD \&CAL with sCD14 level of chronic periodontitis groups.

\section{Discussion}

In the present study, a non-significant statistical difference among the three chronic periodontitis groups for PLI. It was clearly shown that severe group presents the higher mean PLI. This could be due to that the epithelium provides a physical barrier to impede the ingress of bacteria and their products and disruption of the epithelial barrier can lead to further bacterial invasion and inflammation ${ }^{(1)}$, which results in more destruction and break down of the supporting periodontal tissue (either directly by bacterial toxins or indirectly by host immune response), leading to increase the exposed surface area for plaque accumulation on teeth surfaces and increase in the periodontal disease severity. As for GI, a non-significant statistical difference among the chronic periodontitis groups, although severe group presents the higher mean GI. This could be related to the increase in the plaque as the plaque is the causative factor of gingival inflammation. For BOP index results revealed a highly significant difference among the three chronic periodontitis groups, with higher percentage of bleeding sites in the severe group followed by moderate group and lastly the mild group. These findings may indicate the effect of plaque accumulation on blood circulation \& the actual pathophysiological process that happened more in inflamed tissue. And the severity of bleeding \& the ease of its provocation 
depend on the intensity of the inflammation. ${ }^{(\mathbf{1})}$ For PPD A non-significant statistical difference was observed among the chronic periodontitis groups with severe group presented the higher mean among the three groups. This could be due to increase in the bacterial invasion and the amount of plaque that caused destruction of the sulcular \& junctional epithelium \& surrounding alveolar bone. On the other hand regarding CAL,a highly significant difference was observed among the chronic periodontitis groups. It was clearly shown that severe group presents the higher mean among the three groups followed by moderate group and lastly the mild group. This can be explained by the early concepts assumed that after the initial bacterial attack, periodontal tissue destruction continued to be linked to bacterial action. More recently, it was established that the host's immunoinflammatory response to the initial \& persistent bacterial attack unleashes mechanisms that lead to collagen \& bone destruction. These mechanisms are related to various cytokines, some produced normally by cells in non-inflamed tissue and others by cells involved in the inflammatory process such as PMNs, monocytes $\&$ other cells, leading to collagen $\&$ bone destruction. ${ }^{(1)}$

In present study, sCD14 salivary levels were elevated in chronic periodontitis groups\& higher than in control group. And further establish sCD14 as an acute phase protein in periodontitis, whose level increases with disease severity. Many functions have been attributed to acute-phase proteins, including tissue repair, modulation of coagulation, neuroendocrine secretion, bacterial opsonization \& clearing, hemopoiesis, metal binding, and, in the case of CD14, fighting infection. ${ }^{(24)}$ Regarding the sCD14 level for the study groups, a highly significant result in sCD14 level was shown among the three chronic periodontitis groups.Inter groups' comparison revealed a statistical significant difference between the mild \& moderate groups \& a highly significant difference between the mild \& severe groups. A non-significant difference was found between the moderate $\&$ severe groups. This result was in agreement with other study, ${ }^{(\mathbf{2 9})}$ in their study, although the samples analyzed were different, the levels of sCD14 did not differ significantly between the periodontitis groups. Similar results have also been reported by other study. ${ }^{\mathbf{( 1 5})}$ Also this result is in agreement with earlier study, ${ }^{(24)}$ who evaluated the sCD14 levels in GCF by immunoblotting. While limited information is available on the exact contribution of sCD14 to, \& its mechanism of action in, the pathogenesis of periodontal disease, it can be speculated that sCD14 plays a significant role because it is detected in elevated amounts in the GCF of patients with periodontitis. ${ }^{(24)}$

For the clinical-immunological correlations, there were weak correlation with PLI, GI and BOP parameters. This result may be due to small number of samples and there are no data to compare the results with it .

Regarding the correlation between the PPD parameters and the sCD14 level we noticed a positive highly significant correlation between the PPD and CAL parameters with the SCD14 level. This might be interpreted as more production of sCD14 in cases with mild-to- moderate -to- severe periodontal breakdown, which is consistent with the deleterious role of sCD14 because of LPS potent stimulation of sCD14 release (29) and shedding from monocytes/macrophages ${ }^{\mathbf{3 0})}$ and activated neutrophils. As shedding implies the release of the ectodomain of a cell-surface molecule that will keep its biological activity, ${ }^{(24)}$ once present in the extracellular environment in a soluble and biologically active form, sCD14 can interact with cells lacking cell-surface CD14 such as endothelial and epithelial cells. ${ }^{(21}$, ${ }^{31)}$ Thus, sCD14 could mediate cell activation induced by endotoxin and whole bacteria, resulting in the production of a potent immune response and proinflammatory mediators, ${ }^{(32)}$ and amplifying the inflammatory process, ${ }^{(21 ; 31)}$ which further take part in the tissue destruction and bone resorption observed in periodontitis. ${ }^{(\mathbf{3 3})}$

In addition, more evidence suggests that when bacteria propagate in the periodontal pocket, salivary sCD14 promotes their invasion and induces production of IL-8 by oral epithelial cells to recruit neutrophils and T-cells and activate neutrophils for the initiation and establishment of an innate immune response to the bacteria at the site of infection. ${ }^{(34)}$

\section{References}

[1]. Carranza, Newman, Takei \&Klokkevold. Carranza's Clinical Periodontology, $11^{\text {th }}$ Edition, 2012. Elsevier, Saunders.

[2]. Hinrichs JE \& Novak MJ. Carranza's Clinical Periodontology (Chapter 4), $11^{\text {th }}$ Edition, 2012. Elsevier, Saunders.

[3]. American Academy of Periodontology. Parameter on Chronic Periodontitis with Advanced Loss of Periodontal Support and Parameter on Chronic Periodontitis with Slight to ModerateLoss of Periodontal Support (Supplements). J Periodontol, May 2000.

[4]. Wurfel MM \& Wright SD. LBP \& Soluble CD14 Transfer LPS to Phospholipid Bi-Layers: Preferential Interaction with Particular Classes of Lipid. J. Immunol., 1997: 158: 3925-3934.

[5]. Sugiyama T \& Wright SD. Soluble CD14 Mediates Efflux of Phospholipids From Cells. J. Immunol., 2001: 166: 826-831.

[6]. Haziot A, Chen S, Ferrero E, Low MG, Silber R \&Goyert SM et al. The Monocyte Differentiation Antigen, CD14, Is Anchored to the Cell Membrane by a Phosphatidylinositol Linkage. J Immunol, 1988: 141: 547-552.

[7]. Lin B, Noring R, Steere AC, Klempner MS \& Hu LT et al. Soluble CD14 Levels in the Serum, Synovial Fluid, \& Cerebrospinal Fluid of Patients with Various Stages of Lyme disease. J Infect Dis, 2000: 181: 1185-1188.

[8]. Hatakeyama J, Tamai R, Sugiyama A, Akashi S, Sugawara S \& Takada H et al. Contrasting Responses of Human Gingival \& Periodontal Ligament Fibroblasts to Bacterial Cell-Surface Components through the CD14/TLR System. Oral Microbiol Immunol, 2003: 18: 14-23

[9]. Bazil V, Baudys M \& Hilgert I et al. Structural Relationship between the Soluble \& Membrane-bound Forms of Human Monocyte Surface Glycoprotein CD14. MolImmunol, 1989: 26: 657-662.

[10]. Wright SD, Ramos RA, Tobias PS, Ulevitch RJ \&Mathison JC et al. CD14, A Receptor for Complexes of LPS \& LBP. Science, 1990: 249: 1431-1433. 
[11]. Bazil V, Horejsi V \& Baudys M et al. Biochemical Characterization of a Soluble Form of the 53-kDa Monocyte Surface Antigen. Eur J Immunol, 1986: 16: 1583-1589.

[12]. Jin L \& Darveau RP. Soluble CD14 Levels in GCF of Subjects with Untreated Adult Periodontitis. J Periodontol, 2001: 72: 634640 .

[13]. Bussolati B, David S, Cambi V, Tobias PS \&Camussi G et al. Urinary Soluble CD14 Mediates Human Proximal Tubular Epithelial Cell Injury Induced by LPS. Int J Mol Med, 2002: 10: 441-449.

[14]. Duncan L, Yoshioka M, Chandad F \&Grenier D et al. Loss of LPS Receptor CD14 from the Surface of Human Macrophage-like Cells Mediated by P. gingivalis Outer Membrane Vesicles. Microb Pathog, 2004: 36: 319-325.

[15]. Isaza-Guzman DM, Aristizabal-Cardona D, Martinez-Pabon MC, Velasquez-Echeverri H \&Tobon-Arroyave SI et al. Estimation of sCD14 Levels in Saliva Obtained from Patients with Various Periodontal Conditions. Oral Dis, 2008: 14: $450-456$.

[16]. Kirkland TN \& Viriyakosol S. Structure-Function Analysis of Soluble \& Membrane-bound CD14. Prog. Clin. Biol. Res., 1998:397: 79-87.

[17]. Antal-Szalmas P, Szollosi I, Lakos G, Kiss E, Csipo I, Sumegi A, Sipka S, Jos A.G. van Strijp, Kok P.M. van Kessel\&GyulaSzegedi G et al. A Novel Flow Cytometric Assay to Quantify Soluble CD14 Concentration in Human Serum. Cytometry, 2001: 45:115-123.

[18]. Haziot A, Tsuberi BZ \&Goyert SM et al. Neutrophil CD14: Biochemical Properties \& Role in the Secretion of TNF- $\alpha$ in Response to LPS. J Immunol, 1993: 150: 5556-5565.

[19]. Pugin J, Schurer-Maly CC, Leturcq D, Moriarty A, Ulevitch RJ \& Tobias PS et al. LPS Activation of Human Endothelial \& Epithelial Cells is mediated by LBP \& Soluble CD14. ProcNatlAcadSci USA, 1993: 90: 2744-2748.

[20]. Nicu EA, Laine ML, Morre SA, Van der Velden U \& Loos BG et al. Soluble CD14 in Periodontitis. Innate Immun, 2009: 15: 121128.

[21]. Frey EA, Miller DS \& Jahr TG et al. Soluble CD14 Participates in the Response of Cells to LPS. J Exp Med, $1992: 176: 1665-1671$.

[22]. Backhed F, Meijer L, Normark S \& Richter-Dahlfors A et al. TLR4-dependent Recognition of LPS by Epithelial Cells Requires sCD14. Cell Microbiol, 2002: 4: 493-501.

[23]. Feghali K, Tanabe S \&Grenier D et al. sCD14 Induces Cytokine Release by Human Oral Epithelial Cells. J Periodont Res, 2011: 46: 147-152.

[24]. Bas S, Gauthier BR, Spenato U, Stingelin S \&Gabay C et al. CD14 is An Acute-Phase Protein. J Immunol, 2004: 172: 4470-4479.

[25]. Rossa C Jr \& Kirkwood K. Carranza's Clinical Periodontology (Chapter 25), $11^{\text {th }}$ Edition, 2012. Elsevier, Saunders.

[26]. Bainbridge BW, Coats SR, \& Darveau RP et al.P.gingivalis LPS Displays Functionally Diverse Interactions with the Innate Host Defense System. Ann Periodontol: Vol. 7, No. 1, Dec 2002.

[27]. Rey Nores JE, Bensussan A \& Vita N et al. Soluble CD14 Acts as a Negative Regulator of Human T-Cell Activation \& Function. Eur J Immunol, 1999: 29: 265-276.

[28]. Arias MA, Rey Nores JE \& Vita N et al. Cutting Edge: Human B-cell Function is regulated by Interaction with Soluble CD14: Opposite Effects on IgG1 \&IgE Production. J Immunol, 2000: 164: 3480-3485.

[29]. Hayashi J, Masaka T \& Ishikawa I et al. Increased Levels of Soluble CD14 in Sera of Periodontitis Patients. Infect Immun, 1999: 67: 417-420.

[30]. Bazil V \& Strominger JL. Shedding as a Mechanism of Down-modulation of CD14 on Stimulated Human Monocytes. J Immunol, 1991: 147: 1567-1574.

[31]. Schumann RR \& Rietschel ET. The role of CD14 \& LBP in the Activation of Different Cell Types by Endotoxin. Med Microbiol Immunol, 1994: 183: 279-297.

[32]. Labeta MO, Vidal K, Rey Nores JE et al. Innate Recognition of Bacteria in Human Milk is mediated by a Milk-derived Highly Expressed Pattern Recognition Receptor, soluble CD14. J Exp Med, 2000: 191: 1807-1812.

[33]. Page RC. The Role of Inflammatory Mediators in the Pathogenesis of Periodontal Disease. J Periodontal Res, 1991: 26: 230-242.

[34]. Takayama A, Satoh A \& Ngai T et al. Augmentation of Actinobacillus actinomycetemcomitans Invasion of Human Oral Epithelial Cells \& Up-regulation of IL-8 Production by Saliva CD14. Infect Immun, 2003: 71: 5598-5604.

Table (1): The Mean Values of PLI, GI \& the Percentages of Sites with BOP among the chronic periodontitis Groups

\begin{tabular}{|c|c|c|c|c|c|c|c|}
\hline & & Mild & Moderate & Severe & $\mathbf{F}$ & p-value & Sig \\
\hline \multirow{2}{*}{ PLI } & Mean & 1.3947 & 1.1987 & 1.4400 & \multirow{2}{*}{1.219} & \multirow{2}{*}{0.306} & \multirow{2}{*}{ NS } \\
\hline & \pm SD & 0.58696 & 0.28807 & 0.42396 & & & \\
\hline \multirow{2}{*}{ GI } & Mean & 1.2613 & 1.1293 & 1.4120 & \multirow{2}{*}{2.242} & \multirow{2}{*}{0.119} & \multirow{2}{*}{ NS } \\
\hline & \pm SD & 0.41845 & 0.25524 & 0.40154 & & & \\
\hline \multirow{2}{*}{ BOP } & Score 0 & $58.2 \%$ & $41.6 \%$ & $25.3 \%$ & \multirow{2}{*}{ Chi 314.972} & \multirow{2}{*}{0.000} & \multirow{2}{*}{ HS } \\
\hline & Score 1 & $41.8 \%$ & $58.4 \%$ & $74.7 \%$ & & & \\
\hline
\end{tabular}

Table (2): Inter Group Comparison among the chronic periodontitis Groups of the Percentages of Sites with BOP

\begin{tabular}{|c|c|c|c|c|}
\hline \multirow{4}{*}{ BOP } & Inter Group Comparison & Chi & p-value & Sig. \\
\cline { 2 - 5 } & Mild x Moderate & 79.685 & 0.000 & HS \\
\cline { 2 - 5 } & Mild x Severe & 313.573 & 0.000 & HS \\
\cline { 2 - 5 } & Moderate x Severe & 80.845 & 0.000 & HS \\
\hline
\end{tabular}

Table (3): The Mean Values of PPD \& CAL among the chronic periodontitis Groups

\begin{tabular}{|c|c|c|c|c|c|c|c|}
\hline & & Mild & Moderate & Severe & $\mathbf{F}$ & p-value & Sig. \\
\hline \multirow{2}{*}{ PPD } & Mean & 4.50933 & 4.64913 & 4.89053 & \multirow{2}{*}{2.831} & \multirow{2}{*}{0.070} & \multirow{2}{*}{ NS } \\
\hline & $\pm \mathrm{SD}$ & 0.451655 & 0.404584 & 0.472738 & & & \\
\hline \multirow{2}{*}{ CAL } & Mean & 2.42480 & 2.86413 & 4.69493 & \multirow{2}{*}{45.965} & \multirow{2}{*}{0.000} & \multirow{2}{*}{ HS } \\
\hline & $\pm \mathrm{SD}$ & 0.614723 & 0.557106 & 0.855003 & & & \\
\hline
\end{tabular}


Table (4): Inter Groups Comparison among the chronic periodontitis Groups of the Mean of CAL

\begin{tabular}{|c|c|c|c|c|}
\hline Inter Group Comparison & t-test & Mean Difference & $\boldsymbol{p}$-value & Sig. \\
\hline Mild x Moderate & 2.051 & 0.439333 & 0.050 & $\mathrm{~S}$ \\
\hline Mild x Severe & 8.349 & 2.270133 & 0.000 & $\mathrm{HS}$ \\
\hline Moderate x Severe & 6.948 & 1.830800 & 0.000 & $\mathrm{HS}$ \\
\hline
\end{tabular}

Table (5) The Median of sCD14 Level in the Control \& Chronic Periodontitis Groups

\begin{tabular}{|c|c|c|c|c|c|}
\hline & Groups & Median & Z-value & p-value & Sig. \\
\hline $\begin{array}{c}\text { sCD14 } \\
\text { Level }\end{array}$ & Control & 5.428 & 4.587 & 0.000 & HS \\
\cline { 2 - 3 }
\end{tabular}

Table (6) Inter Group Comparison of sCD14 Level between the Control Group\& the chronic periodontitis Groups

\begin{tabular}{|c|c|c|c|}
\hline Inter Groups Comparison & Z-value & p-value & Sig. \\
\hline Control x Mild & 2.678 & 0.007 & HS \\
\hline Control x Moderate & 4.592 & 0.000 & $\mathrm{HS}$ \\
\hline Control x Severe & 3.896 & 0.000 & $\mathrm{HS}$ \\
\hline & & & \\
\hline
\end{tabular}

Table (7) The Median of sCD14 Level among the chronic periodontitis Groups

\begin{tabular}{|c|c|c|c|c|c|c|c|}
\hline & Groups & No. & Median & Chi & $d f$ & -value & Sig. \\
\hline \multirow{2}{*}{$\begin{array}{c}\text { sCD14 } \\
\text { Level }\end{array}$} & Mild & 15 & 7.392 & & & & \\
\cline { 2 - 5 } & Moderate & 15 & 10.359 & \multirow{2}{*}{10.985} & 2 & 0.004 & HS \\
\cline { 2 - 5 } & Severe & 15 & 12.798 & & & \\
\hline
\end{tabular}

Table (8) Inter Group Comparison of sCD14 Level among the chronic periodontitis Groups

\begin{tabular}{|c|c|c|c|}
\hline Inter Groups Comparison & Z-value & $p$-value & Sig. \\
\hline Mild x Moderate & 2.306 & 0.021 & S \\
\hline Mild x Severe & 2.937 & 0.003 & HS \\
\hline Moderate x Severe & 1.668 & 0.095 & NS \\
\hline
\end{tabular}

Table (9) Correlation between the Periodontal Parameters \& the sCD14 Level among the chronic periodontitis Groups

\begin{tabular}{|c|c|c|c|c|c|c|c|c|c|c|}
\hline \multirow{2}{*}{$\begin{array}{c}\text { sCD14 } \\
\text { Level }\end{array}$} & \multicolumn{2}{|c|}{ PLI } & \multicolumn{2}{|c|}{ GI } & \multicolumn{2}{|c|}{ BOP } & \multicolumn{2}{|c|}{ PPD } & \multicolumn{2}{|c|}{ CAL } \\
\hline & $r$ & p-value & $r$ & p-value & $r$ & p-value & $r$ & p-value & $r$ & p-value \\
\hline Mild & 0.315 & 0.253 & 0.103 & 0.715 & 0.257 & 0.356 & 0.444 & 0.097 & 0.405 & 0.134 \\
\hline Moderate & 0.274 & 0.323 & 0.375 & 0.168 & 0.084 & 0.766 & 0.132 & 0.640 & 0.375 & 0.168 \\
\hline Severe & 0.441 & 0.100 & 0.133 & 0.637 & 0.209 & 0.454 & 0.046 & 0.870 & 0.055 & 0.844 \\
\hline
\end{tabular}

Table (10) Correlation between PPD \& CAL Means of Chronic Periodontitis Group with the sCD14 Level

\begin{tabular}{|c|c|c|c|}
\hline \multicolumn{2}{|c|}{} & PPD & CAL \\
\hline \multirow{2}{*}{ sCD14 } & $r$ & 0.489 & 0.504 \\
\cline { 2 - 4 } Level & $p$-value & 0.001 & 0.000 \\
\hline
\end{tabular}

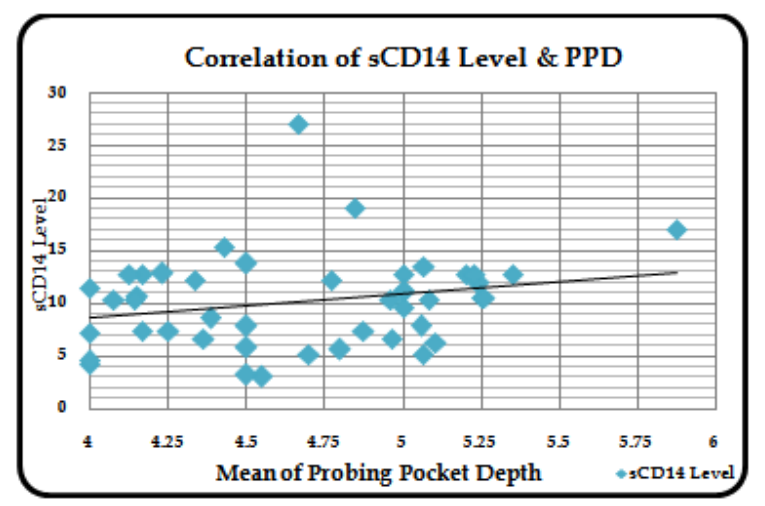

Figure (1) Scatter Plot Showing the Linear Correlation between PPD with sCD14 Level of Chronic Periodontitis Group, Each Symbol Represents One Subject 


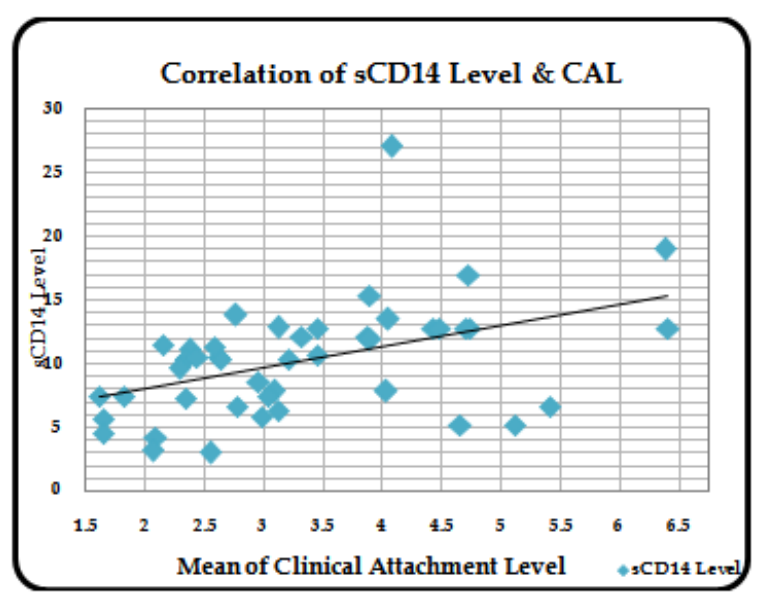

Figure (2) Scatter Plot Showing the Linear Correlation between CAL with SCD14 Level of Chronic Periodontitis Group, Each Symbol Represents One Subject 